\title{
The Properties of Shuffle Screw Dislocations in Semiconductors Silicon and Germanium
}

\author{
Huili Zhang ${ }^{*}$, Chun Zhang, Chunhua Zeng and Lumei Tong
}

Kunming University of Science and Technology, Kunming 650093, P.R. China

\begin{abstract}
The dislocation widths, Peierls barriers and Peierls stresses for shuffle screw dislocations in diamond structure crystals, Si and Ge, have been calculated by the improved P-N theory. The widths are about $0.6 \mathrm{~b}$, where $\mathrm{b}$ is the Burgers

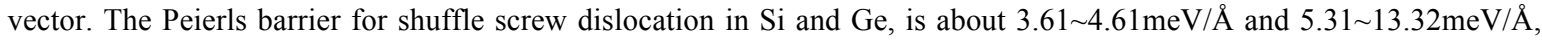
respectively. The Peierls stress is about $0.28 \sim 0.33 \mathrm{GPa}$ and $0.31 \sim 0.53 \mathrm{GPa}$, respectively. The calculated Peierls barriers and stresses are likely the results of shuffle screw dislocation with metastable core which is centered on the bond between two atoms.
\end{abstract}

Keywords: Dislocation width, Peierls barrier, Peierls stress, Shuffle screw dislocation.

\section{INTRODUCTION}

The mechanical properties of Si and Ge have been widely investigated due to their importance in the electronic industry. The plasticity of crystal materials is closely related to the dynamic properties of dislocations. The dislocations in diamond structure crystals $\mathrm{Si}$ and Ge can be present in the glide set or shuffle set configurations and the glide dislocation will dissociate into a pair of partial dislocations separated by an intrinsic stacking fault [1]. In experiments, Rabier and Demenet have showed that high external pressures on Si favor a pure shuffle dislocation popular over the partialized glide dislocation [2]. In order to make better use of semiconductors $\mathrm{Si}$ and $\mathrm{Ge}$, studying the structure and motion of shuffle dislocation is important. Several attempts have been made to determine the Peierls stress of the shuffle $60^{\circ}$ dislocations. However, the screw dislocation has been less studied. $A b$ initio calculations carried out by Cai et al. predicted the Peierls stress at zero pressure for $\mathrm{Si}$ to be $3.3 \pm 0.2 \mathrm{GPa}$ [3]. Density functional theory carried out by Pizzagalli et al. gives the result $4.1 \pm 0.3 \mathrm{GPa}$ [4].

Besides the numerical methods, the analytical P-N theory $[1,5,6]$ is generally used for studying the structure and motion properties of dislocations. However, because of treating the crystal as an elastic continuum body, the classical P-N model becomes increasingly inaccurate for narrow dislocations $[5,7,8]$. Recently, professor Wang have obtained the improved P-N equation which has relaxed the continuum approximation successfully [9-11]. The research results have shown that the improved P-N theory can remarkably improve the agreement between theoretical prediction and the numerical result $[12,13]$.

*Address correspondence to this author at the Kunming University of Science and Technology, Kunming 650093, P.R. China; Tel: 18669218102 ; E-mail: zh1010195@163.com
In this paper, the core structure, Peierls barrier and Peierls stress for shuffle screw dislocations in $\mathrm{Si}$ and Ge have been studied by the improved P-N theory. An overview of this paper is as follows: Sec 1, Introduction; Sec 2, Dislocation equation, core structure, Peierls barrier and Peierls stress; Sec 3 is the result and discussion. The last section 4 is the conclusion.

\section{DISLOCATION EQUATION, CORE STRUCTURE, PEIERLS BARRIER AND PEIERLS STRESS}

Based on the lattice dynamics and the symmetry principle, the improved P-N equation for the straight dislocation that describes the balance of atoms on the border can be expressed as:

$$
-\frac{\beta}{2} \frac{d^{2} u(\mathrm{x})}{d x^{2}}-\left.\frac{K \sigma}{2 \pi} \int_{-\infty}^{+\infty} \frac{d x^{\prime}}{x^{\prime}-x}\left(\frac{d u}{d x}\right)\right|_{x=x}=f(u)
$$

where $u$ is the displacement field, $f(u)$ is the restoring force, and they are defined along the Burgers vector. $\sigma$ is the area of primitive cell in the misfit plane. The discrete parameter $\beta$ and energy factor $\mathrm{K}$ can be represented as $[1,14]$ :

$$
\begin{aligned}
& \beta=\left(\frac{3 c_{11}+5 c_{12}}{24} \sin ^{2} \theta+\frac{c_{11}-c_{12}}{16} \cos ^{2} \theta\right) \mathrm{a}_{0}^{3}, \\
& K=\left(\frac{\sin ^{2} \theta}{1-v}+\cos ^{2} \theta\right) \mu
\end{aligned}
$$

Where $\theta$ is the dislocation angel, $\mu$ and $v$ are the effective shear modulus and Poisson's ratio within $\{111\}$ plane [1,5], $c_{11}$ and $c_{12}$ are the elastic constants, and $a_{0}$ is the lattice constant. The values for these constants are listed in Table $\mathbf{1}$. 
Table 1. The effective shear modulus and Poisson's ratio $\mu$ and $v$ within $\{111\}$ plane, elastic constants $c_{11}$ and $c_{12}$, and the lattice constant $a_{0}$ for Si and Ge, where $\mu, c_{11}$ and $c_{11}$ are in units of GPa, $a_{0}$ in unit of $\AA$.

\begin{tabular}{|c|c|c|c|c|c|}
\hline & $\mu$ & $v$ & $c_{11}$ & $c_{12}$ & $a_{0}$ \\
\hline \hline $\mathrm{Si}$ & 63.75 & 0.256 & 165.7 & 63.9 & 5.43 \\
\hline $\mathrm{Ge}$ & 52.00 & 0.248 & 128.9 & 48.3 & 5.65 \\
\hline
\end{tabular}

Table 2. The modification factors $\Delta_{1}$ and $\Delta_{2}$. SW and Baskes represent the $\gamma$-surface that has been calculated by Stillinger-Weber and MEAM-Baskes inter-atomic potential, respectively; LDA and GGA represent the $\gamma$-surface that has been calculated by Vienna ab initio simulation package (VASP) with the local density approximation and the generalized gradient approximation, respectively.

\begin{tabular}{|c|c|c|c|c|}
\hline & Si & & Ge & $\Delta_{2}$ \\
\hline \hline & $\Delta_{1}$ & $\Delta_{2}$ & $\Delta_{1}$ & 0.26 \\
\hline SW & -0.82 & 0.315 & -0.40 & -0.38 \\
\hline Baskes & -0.10 & -0.16 & -1.10 & 0.84 \\
\hline LDA & -1.00 & 0.76 & -1.20 & 0.90 \\
\hline GGA & -0.90 & 0.68 & & \\
\hline
\end{tabular}

The restoring force $f(u)$ in Eq.(1) is given by the gradient of the $\gamma$-surface [15]

$f=-\nabla \gamma(\mathrm{u}) \sigma$

The $\gamma$-surface of shuffle set for Si and Ge has been calculated by Kang and Cai [16], and it can be expressed as follows [17]:

$$
\begin{aligned}
\gamma_{b}(\mathrm{u})= & \frac{\mu b^{2}}{4 \pi^{2} d}\left(1+\cos \frac{2 \pi u}{b}\right)\left(1+\Delta_{1} \cos ^{2} \frac{\pi u}{b}\right. \\
& \left.+\Delta_{2} \cos ^{4} \frac{\pi u}{b}\right),
\end{aligned}
$$

where $b$ and $d$ are the Burgers vector and the spacing between glide planes, respectively. $\Delta_{1}$ and $\Delta_{2}$ are the modification factors to the sinusoidal-force law. For fitting the $\gamma$ surface given in Ref. [16], $\Delta_{1}$ and $\Delta_{2}$ have been listed in Table 2. The $\gamma$-surface and the fitted curve have been plotted in Fig. (1).

The dislocation Eq.(1) can be solved by truncating method proposed by professor Wang and the trial solution possesses the following form $[14,18]$

$u=\frac{b}{\pi}\left(\arctan q+\frac{c q}{1+q^{2}}\right)$,

with

$q=k x, k=k_{0}(1-\mathrm{c}), \mathrm{k}_{0}=\frac{2}{d}\left(\frac{\sin ^{2} \theta}{1-v}+\cos ^{2} \theta\right)^{-1}$,

where the parameter $c$ is a constant that can be determined by the dislocation equation.
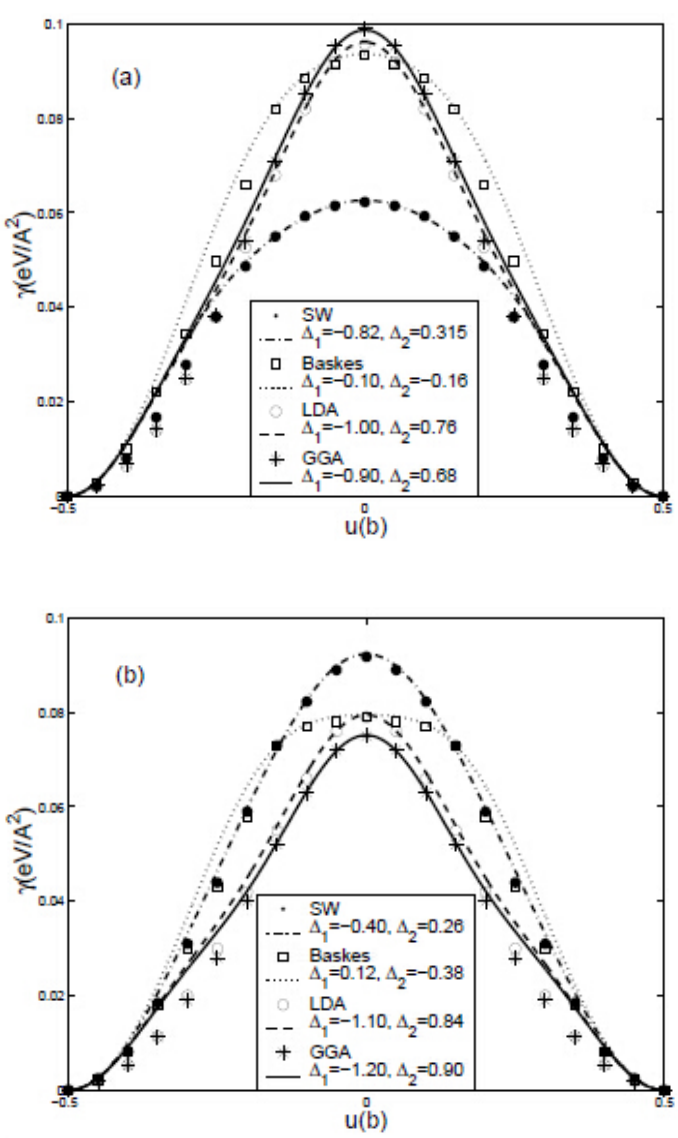

Fig. (1). The $\gamma$-surface along $<110>$ direction of shuffle set for $\mathrm{Si}$ and Ge given by Kang et al. [16] and fitted by Eq. (3), where the Burgers vectors for $\mathrm{Si}$ and $\mathrm{Ge}$ are $3.84 \AA$ and $4.00 \AA$, respectively. 
Table 3. The core parameter $c$ and half width $\xi . c_{0}$ and $\xi_{0}$ are given by the classical P-N model.

\begin{tabular}{|c|c|c|c|c|c|}
\hline & Potential & $c_{0}$ & $c$ & $\xi_{0}(b)$ & $\xi(b)$ \\
\hline & SW & 0.85 & 0.88 & 0.99 & 1.21 \\
\hline \multirow[t]{4}{*}{$\mathrm{Si}$} & Baskes & 0.62 & 0.76 & 0.46 & 0.66 \\
\hline & LDA & 0.48 & 0.73 & 0.38 & 0.60 \\
\hline & GGA & 0.46 & 0.72 & 0.37 & 0.58 \\
\hline & SW & 0.39 & 0.69 & 0.35 & 0.54 \\
\hline \multirow[t]{3}{*}{$\mathrm{Ge}$} & Baskes & 0.63 & 0.76 & 0.47 & 0.66 \\
\hline & LDA & 0.50 & 0.74 & 0.38 & 0.62 \\
\hline & GGA & 0.57 & 0.76 & 0.42 & 0.66 \\
\hline
\end{tabular}

Substituting the solution Eq.(4) into dislocation equation and after complicated calculations, an algebraic equation about parameter $c$ can be obtained as follows:

$$
\frac{2 \beta \mu}{K^{2} \sigma d}(1+2 \mathrm{c})(1-\mathrm{c})^{2}-\frac{4 c^{2}}{5}-\Delta_{1}\left(1+\frac{c}{5}\right)-\frac{6 \Delta_{2}}{5}=0 .
$$

It recovers the classical P-N model when the discrete parameter $\beta$ equals to zero.

The core parameter $c$ calculated from Eq.(6) and half width (the distance that $u$ changes from 0 to $b / 4$ ) are listed in Table 3 .

In the classical P-N theory, the Peierls barrier and stress are obtained by calculating misfit energy only. However, it has been shown that the contributions of strain energy and misfit energy are equally important [19]. The total energy which includes the contribution of misfit and strain energies should be evaluated to obtain the correct Peierls barrier and Peierls stress. For a dislocation with length $L$, the strain and misfit energies of dislocation per unit length are given by [14].

$$
\begin{aligned}
& E_{s t r}\left(x_{0}\right)=\frac{1}{2} \sum_{l=-\infty}^{\infty} f_{b}\left(u_{l}\right) u_{l} \times \frac{\sigma}{a}, \\
& E_{m i s}\left(x_{0}\right)=\sum_{l=-\infty}^{\infty} \gamma_{b}\left(u_{l}\right) \times \frac{\sigma}{a},
\end{aligned}
$$

where $u_{1}=u\left(x_{1}-x_{0}\right)$ is the relative displacement for dislocation located at $x_{0}, a$ is the length of the primitive vector (period in the direction of dislocation line). Just as shown in Fig. (2), sum is carried over the atoms located in the horizontal band in the misfit plane (the band width is $a$ ). According to Eq.(7) and Eq.(8), the total energy is:

$$
\begin{aligned}
& E_{\text {tot }}=\sum_{l=-\infty}^{\infty} \frac{\mu b^{2} \sigma}{4 \pi^{2} a d}\left\{( 1 + \operatorname { c o s } \frac { 2 \pi u _ { l } } { b } ) \left(1+\Delta_{1} \cos ^{2} \frac{\pi u_{l}}{b}\right.\right. \\
& \left.+\Delta_{2} \cos ^{4} \frac{\pi u_{l}}{b}\right)+\left[\left(1+\Delta_{1}\right) \sin \frac{2 \pi u_{l}}{b}+\frac{\Delta_{1}}{2} \sin \frac{4 \pi u_{l}}{b}\right. \\
& \left.\left.+3 \Delta_{2} \sin \frac{2 \pi u_{l}}{b} \cos ^{4} \frac{\pi u_{l}}{b}\right] \mathrm{u}_{l}\right\} .
\end{aligned}
$$

Due to discreteness of lattice, a dislocation cannot move unless the applied stress exceeds the Peierls stress. The Pei- erls stress can be obtained from the maximum slope of the total energy:

$$
\sigma_{p}=\max \left|\frac{1}{b} \frac{d \mathrm{E}_{t o t}(\mathrm{x})}{d x}\right| \text {. }
$$

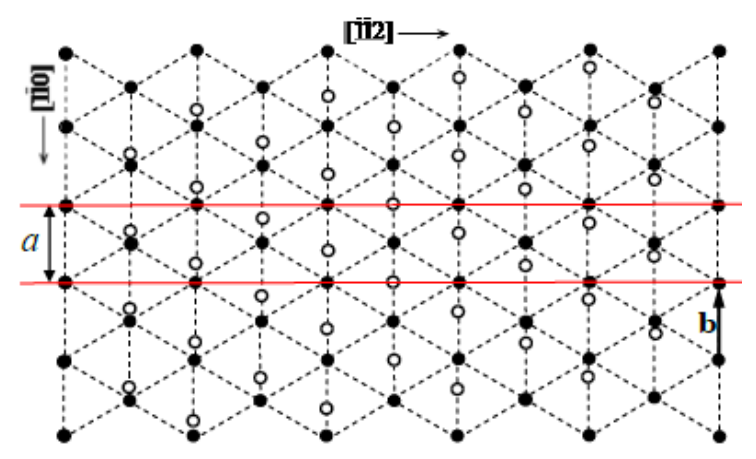

shuffle screw dislocation

Fig. (2). Core structure of the shuffle screw dislocation. The black and white circles represent the atoms on the misfit planes that above and below the cut plane, respectively.

The calculated Peierls barriers and Peierls stresses are listed in Table 4.

\section{RESULT AND DISCUSSION}

The width of dislocation is mainly related to the unstable stacking fault energy. The higher the unstable stacking fault energy, the narrower the dislocation is. The widths of shuffle screw dislocations in $\mathrm{Si}$ and $\mathrm{Ge}$ are about $0.6 b$. The Peierls barriers and Peierls stresses calculated from SW potential are much lower than those calculated from three other potentials. Besides, the results given by classical P-N theory calculated from Baskes potential are much lower than those calculated from LDA and GGA potentials. Thus, the results calculated from LDA and GGA potentials are thought to be more reliable. The Peierls barrier for shuffle screw dislocation in $\mathrm{Si}$ and $\mathrm{Ge}$ is $3.61 \sim 4.61 \mathrm{meV} / \AA$ and $5.31 \sim 13.32 \mathrm{meV} / \AA$, respectively. Peierls stress is $0.28 \sim 0.33 \mathrm{GPa}$ and $0.31 \sim 0.53 \mathrm{GPa}$, respectively. The calculated Peierls stress for $\mathrm{Si}$ is about one magnitude lower than the numerical results given in $[3,4]$. The research on shuffle screw dislocation in $\mathrm{Si}$ shows that there exist two different core structures: core A is centered in the 6-member ring of atoms [20], core B is centered on the 
Table 4. The Peierls barrier $(\mathrm{meV} / \AA ̊)$ and Peierls stress $\left(\mathrm{meV} / \AA^{3}\right)$ for shuffle screw dislocation. $E_{\mathrm{p}}(0)$ and $\sigma_{\mathrm{p}}(0)$ are given by classical P-N model.

\begin{tabular}{|c|c|c|c|c|c|}
\hline & Potential & $E_{p}(\boldsymbol{\theta})$ & $E_{p}$ & $\sigma_{p}(\theta)$ & $\sigma_{p}$ \\
\hline & SW & 2.19 & 1.45 & 0.54 & 0.36 \\
\hline \multirow[t]{4}{*}{$\mathrm{Si}$} & Baskes & 13.85 & 6.52 & 4.13 & 1.65 \\
\hline & LDA & 134.69 & 3.61 & 36.80 & 1.73 \\
\hline & GGA & 137.72 & 4.61 & 37.35 & 2.07 \\
\hline & SW & 128.55 & 9.16 & 30.13 & 2.09 \\
\hline \multirow[t]{3}{*}{$\mathrm{Ge}$} & Baskes & 9.70 & 10.13 & 3.47 & 2.40 \\
\hline & LDA & 99.86 & 5.31 & 29.57 & 1.93 \\
\hline & GGA & 100.50 & 13.32 & 24.90 & 3.29 \\
\hline
\end{tabular}

bond between two atoms [21]. Recent ab initio calculations show that core A is the ground state of a perfect screw dislocation, while core $\mathrm{B}$ is metastable, with an energy $0.38 \mathrm{eV} / b$ higher than that of core A [3]. The Peierls barriers and Peierls stresses given in this paper are likely the results of metastable screw dislocations.

\section{CONCLUSION}

The core structures, Peierls barriers and Peierls stresses for shuffle screw dislocations in semiconductors $\mathrm{Si}$ and Ge, have been investigated. Our results indicate that the results calculated from LDA and GGA potentials are more reliable. The Peierls barrier for shuffle screw dislocation in $\mathrm{Si}$ and $\mathrm{Ge}$, is about 3.61 4.61 $\mathrm{meV} / \AA$ and $5.31 \sim 13.32 \mathrm{meV} / \AA$, respectively. Peierls stress is $0.28 \sim 0.33 \mathrm{GPa}$ and $0.31 \sim 0.53$ GPa, respectively. The calculated Peierls barriers and Peierls stresses are likely the results of dislocations with metastable core which is centered on the bond between two atoms. Further studies will be carried out to better understand the mechanical properties of shuffle screw dislocation with different core structures.

\section{CONFLICT OF INTEREST}

The authors confirm that this article content has no conflict of interest.

\section{ACKNOWLEDGEMENTS}

The work is supported by the National Natural Science Foundation of China (Grant No.11104120), the National Natural Science Foundation of China (Grant No.11305079) and the Applied and Basic Research Foundation of Yunnan Province (Grant No.2011FZ049).

\section{REFERENCES}

[1] Hirth JP, Lothe J. Theory of Dislocations. $2^{\text {nd }}$ ed. New York: Wiley 1982; p. 373.

[2] Rabier J, Demenet JL. On a change in deformation mechanism in silicon at very high stress: new evidences. Script Mater 2001; 45(11): 1259-65.
[3] Cai W, Bulatov VV, Chang JP, Li J, Yip S. Dislocation core effects on mobility in dislocations in solids. In: Nabarro FRN, Hirth JP, Eds. Chapter 64. Amsterdam: Elsevier 2004; vol. 12: pp. 1-80.

[4] Pizzagalli L, Beauchamp P. First principles determination of the Peierls stress of the shuffle screw dislocation in silicon. Phil Mag Lett 2004; 84(11): 729-36.

[5] Joos B, Ren Q, Duesbery MS. Peierls-Nabarro model of dislocations in silicon with generalized stacking-fault restoring forces. Phys Rev B 1994; 50: 5890.

[6] Peierls RE. The size of a dislocation. Proc Phys Soc 1940; 52(1): 34.

[7] Joos B, Duesbery MS. The Peierls stress of dislocations: an analytic formula. Phys Rev Lett 1997; 78(2): 266.

[8] Bulatov VV, Kaxiras E. Semidiscrete variational Peierls framework for dislocation core properties. Phys Rev Lett 1997; 78(22): 4221.

[9] Wang SF. Lattice theory for structure of dislocations in a twodimensional triangular crystal. Phys Rev B 2002; 65(9): 094111.

[10] Wang SF. An improvement of the Peierls equation by taking into account the lattice effects. Chin Phys 2005; 14(12): 2575.

[11] Wang SF. A unified dislocation equation from lattice statics. J Phys A: Math Theor 2009; 42(2): 025208.

[12] Wang SF, Liu RP, Wu XZ. The discrete correction of the core structure for the $\langle 100>\{010\}$ edge dislocation in bcc Fe. J Phys Condens Matter 2008; 20(48): 485207.

[13] Wu XZ, Wang SF. On the properties of $<111>\{110\}$ dissociated superdislocation in $\mathrm{B} 2$ structure $\mathrm{YAg}$ and $\mathrm{YCu}$ : Core structure and Peierls stress. Front Mater Sci China 2009; 3: 205.

[14] Wang SF, Zhang HL, Wu XZ, Liu RP. J Phys Condens Matter 2010; 22(2): 205-11.

[15] Christian JW, Vitek V. Dislocations and stacking faults. Rep Prog Phys 1970; 33: 307.

[16] Kang K, Cai W. Brittle and ductile fracture of semiconductor nanowires - molecular dynamics simulations. Philos Mag 2007; 87: 2169-89.

[17] Zhang HL. Calculation of shuffle $60^{\circ}$ dislocation width and Peierls barrier and stress for semiconductors silicon and germanium. Eur Phys J B 2011; 81(2): 179-83.

[18] Wang SF. Dislocation solution in slowly varying approximation. Phys Lett A 2003; 313: 408-11.

[19] Wang SF. Dislocation energy and peierls streaa: a rigorous calculation from form the lattice theory. Chin Phys Soc 2006; 15(6): 13019.

[20] Arias TA, Joannopoulos JD. Ab initio theory of dislocation interactions: from close-range spontaneous annihilation to the long-range continuum limit. Phys Rev Lett 1994; 73: 680.

[21] Koizumi H, Kamimura Y, Suzuki T. Core structure of a screw dislocation in a diamond-like structure. Phil Mag A 2000; 80(3): 609. 